\title{
App Review Series Introduction
}

\author{
George Shih
}

Published online: 17 December 2014

(C) Society for Imaging Informatics in Medicine 2014

Most SIIM members and JDI readers are technology aficionados and have long since adopted mobile devices, including smartphones and tablets. For some, these devices have become their first, if not primary source of information, and perhaps their preferred way to work in many situations. We know from recent data that native mobile apps largely predominate in the usage of these mobile devices [80\% of the time] [1] over mobile Web apps. So, naturally most of us are interested in the selection of mobile apps that are available and are passionate about the apps that we use most often.

We're launching a new series to review health mobile apps, many of which are focused around imaging but not always. Some apps cross multiple health specialties (e.g., EHR apps), and others may be non-medical apps used for one reason or another by our healthcare colleagues (e.g., PDF reader app for articles and eBooks).

The format of our review will try to give our readers a sense of whether or not a particular app will be of value to them and will evaluate its features, usability, etc., which is similar to other app review websites. In addition, we will also try to provide some specific information that may be useful for our readership, who is often experts in healthcare technology who assist their non-expert colleagues with their mobile devices. For example, whether an app can work completely offline is useful, given the spotty wifi connections in many hospitals and the growing BYOD (Bring Your Own Device) trend where these personal devices may have limited Internet access. FDA approval of certain mobile apps may also be of interest when deciding which app to use. The app review series will also appear on http://www.siim.org.

G. Shih $(\bowtie)$

525 E 68th St, Box 141, New York, NY 10065, USA

e-mail: george@cornellradiology.org
We hope that you will enjoy this new app review series, and we plan to review a wide range of useful apps for healthcare by many different reviewers. If you would like to suggest an app for review or would like to participate in the review of an app, please contact us at appreview@siim.org.

Initial App Review

Title: Lung Nodule Followup Guidelines

Reviewer: George Shih, MD

Reviewer Disclosure(s): Nothing to disclose

Reviewer Contact: george@cornellradiology.org

Review Date: 9/30/2014

Figure 1

App Name: Lung Nodule Followup Guidelines

App Developer: RADIOLOGIQ, LLC (Charles T. Lau, MD, MBA and Ahmed H. El-Sherief, MD)

App Developer Website: radiologiq.com

App Price: FREE

Apple App Store URL: https://itunes.apple.com/us/app/ lung-nodule-followup-guidelines/id527797391

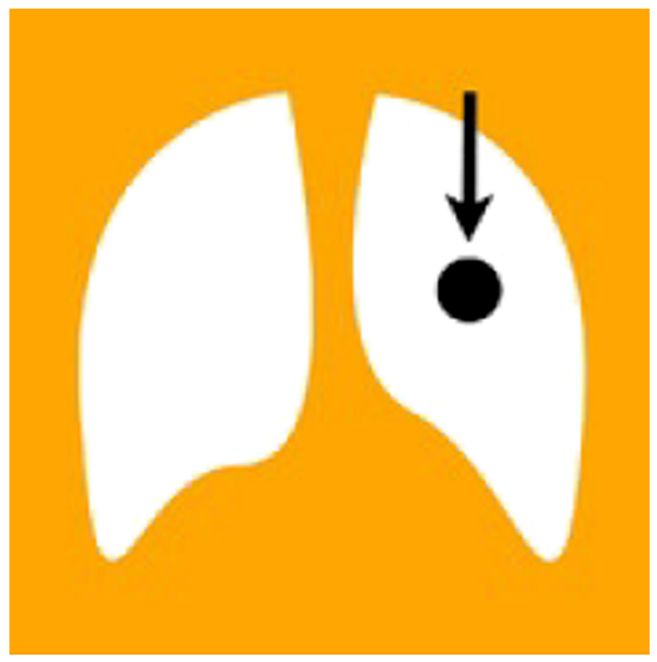

Fig. 1 Lung nodule followup guidelines 
Google Play Store URL: N/A

Category: clinical, reference

Tags: \#reference \#free \#radiology \#lung \#works-offline \#phone-only \#iOS-only

Works Offline: Yes

FDA Approval: N/A

Promotion Code: N/A

\section{Quick Review}

(1 star, lowest; 5 stars, highest)

Overall Rating (1-5): 4.75

Content (1-5): 5

Usability (1-5): 4.5

Pros: Delivers a beautiful and convenient UI for incidental lung nodule follow-up advice based on the Fleishner Society criteria. Offline access (no need for Dr. Google to help)

Cons: Transition between nodule types could be a bit more seamless by saving the nodule size

\section{At A Glance:}

As an abdominal radiologist, I often come across lung bases in CT studies with findings that are straightforward (and occasionally findings that are not). Follow-up for incidental lung nodules in most patients should be straightforward to remember, but for some reason, I have been relying on Google to find the Fleishner Society criteria in table formatuntil now.

The Lung Nodule Followup Guidelines app available for iOS delivers the guidelines with a stunning, easy-to-use format, making my imaging follow-up recommendations a breeze.

\section{Full Review}

\section{Introduction}

As an abdominal radiologist, I often come across lung bases in CT studies with findings that are straightforward (and occasionally findings that are not). Follow-up for incidental lung nodules in most patients should be straightforward to remember, but for some reason, I have been relying on Google to find the Fleishner Society criteria in table formatuntil now.

The Lung Nodule Followup Guidelines app available for iOS delivers the guidelines with an stunning, easy-to-use format, making my imaging follow-up recommendations a breeze.

\section{Purpose/Features/Content:}

The Lung Nodule Followup Guidelines app divides the follow-up guidelines by different lung nodule types (e.g., solid, ground glass, mixed solid and groundglass, etc.). A slide out menu on the left lets you navigated between the different nodule types. Once you select the nodule type, there's a slider for nodule size. The follow-up recommendation is displayed in a circular line graph with time intervals and is also displayed in text format.

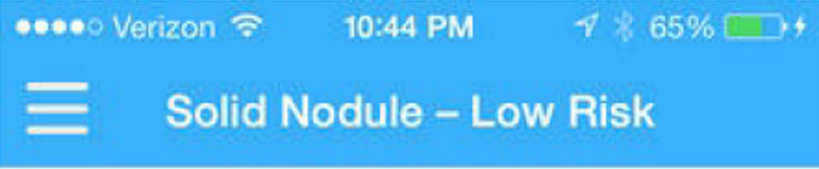

Fleischner Society Follow-Up Recommendation

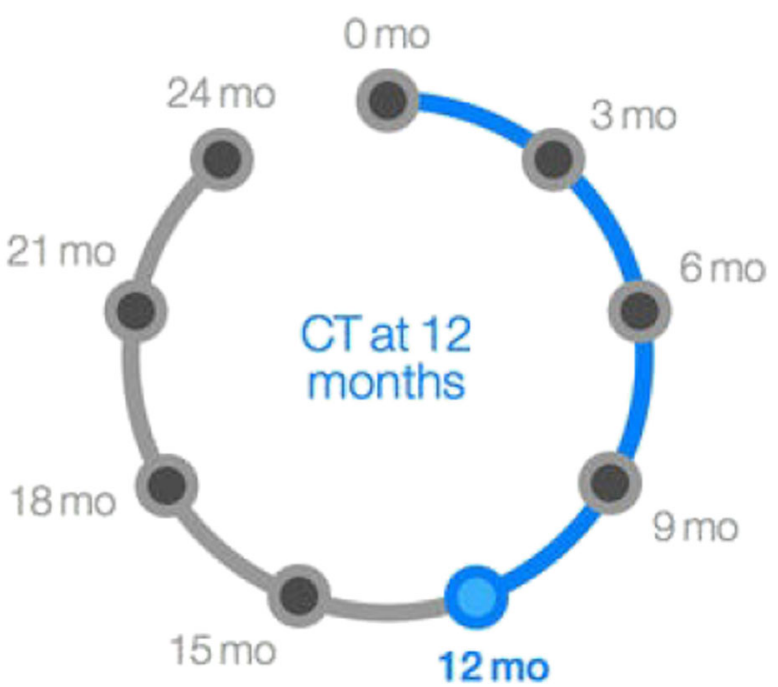

Fig. 2 Follow-up recommendation

There is a good "Notes" section about the guidelines, which may be useful to read the first time a user launches the app, in case they're not familiar with the Fleishner Society criteria (e.g., not useful in patients with known malignancy).

Usability:

The user experience is excellent, with well-chosen colors, and easy-to-understand circular visualization of follow-up time frames. Navigation between nodule types using the slide out menu is also convenient. The only minor issue is that once you've selected a size for a lesion, you can't change your mind about the nodule type (e.g., solid to mixed) without selecting the size again on the slider.

Good: Excellent UI. Good miscellaneous comments about the guidelines, which can really help

Room For Improvement: App doesn't remember the nodule size if you switch to a different nodule type.

I also wish the app would insert the recommendation into my report, but I realize the numerous challenges facing that seemingly small interaction.

\section{$6 \mathrm{~mm}$}

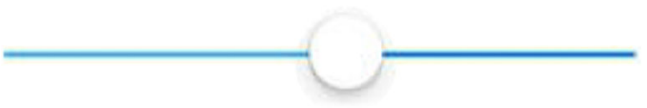

Lung Nodule Diameter

Fig. 3 Nodule diameter 
Figure 2

Figure 3

Similar Apps

Lung Cancer Screening

By RADIOLOGiQ, LLC

Abdominal CT Incidental Finding Guidelines

By RADIOLOGiQ, LLC

Lung Cancer Stage

By Edward Bender
IASLC Staging Atlas in Thoracic Oncology

By Editorial Rx Press

\section{Reference}

1. Venture Beat News. Available at http://venturebeat.com/2013/04/03/ the-mobile-war-is-over-and-the-app-has-won-80-of-mobile-timespent-in-apps/. Accessed 25 November 2014. 